\title{
Precision Full-Wave Rectifier Using Two DDCCs
}

\author{
Montree Kumngern \\ Department of Telecommunications Engineering, Faculty of Engineering, King Mongkut's Institute of Technology \\ Ladkrabang, Bangkok, Thailand \\ E-mail: kkmontre@kmitl.ac.th
}

Received February 22, 2011; revised April 12, 2011; accepted April 19, 2011

\begin{abstract}
A new precision full-wave rectifier employing only two differential difference current conveyors, which is very suitable for CMOS technology implementation, is presented. The proposed rectifier is the voltage-mode circuit, which offers high-input and low-output impedance hence it can be directly connected to load without using any buffer circuits. PSPICE is used to verify the circuit performance. Simulated rectifier results basedon a $0.5 \mu \mathrm{m}$ CMOS technology with $\pm 2.5 \mathrm{~V}$ supply voltage demonstrates high precision rectification and excellent temperature stability. In addition, the application of proposed rectifier to pseudo RMS-to-DC conversion is also introduced.
\end{abstract}

\section{Keywords: Full-Wave Rectifier, Voltage-Mode Circuit, DDCC, RMS-to-DC Conversion}

\section{Introduction}

Full-wave rectifier is used in RF demodulator, piecewise linear function generator, $\mathrm{AC}$ voltmeter, watt meter and various nonlinear analog signal processing circuits. Typically, a conventional rectifier could be realized by using diodes for it rectification. However, this circuit would not be capable of rectifying incoming signals whose amplitudes are less than the threshold voltage (approximately $0.7 \mathrm{~V}$ for silicon diode and 0.3 for germanium diode). As a result, diode-only rectifiers are normally used in only those applications in which the precision in the range of threshold voltage is insignificant, such as RF demodulators and DC voltage supply rectifiers. For high precision applications, the diode-only rectifier cannot be used. This can be overcome by using integrated circuit rectifiers instead. The precision rectifiers based on operational amplifier (op-amp), diodes and resistors are presented [1-4]. However, the classical problem with conventional precision rectifiers based on op-amps and diodes is that during the non-conduction/conduction transition of the diodes, the op-amps must recover with a finite small-signal, $\mathrm{dv} / \mathrm{dt}$, (slew-rate) resulting in significant distortion during the zero crossing of the input signal. The use of the high slew-rate op-amps does not solve this problem because it is a small signal transient problem. The gain-bandwidth is a parameter of op-amp that limits the high frequency performance of this scheme. Moreover, since these structures use the op-amp and the resistors; therefore these circuits are not suitable for IC fabrication. Second-generation current conveyors (CCIIs) is possessed a very high slew rate and bandwidth if compared to the traditional op-amp. This makes the CCII of primary importance in the design of modern analog integrated circuits.

Several circuits based on CCII for realizing full-wave rectification have been reported in the literature [5-10]. The rectifier circuit in [5-7] employ diodes and resistors in addition to CCIIs. The circuit proposed in [8] employs bipolar current mirrors in addition to a CCII and a number of resistors. The rectifier circuit in [9] employs four CCCIIs and resistors. The circuit proposed in [10] employs two CCII and two MOS transistors. However, the use of resistor makes these circuits not ideal for integration. Recently, Chiu et al. [11] proposed a new current conveyor circuit called the differential difference current conveyor (DDCC). The DDCC has the advantages of both the CCII and the differential difference amplifier (DDA) (such as high input impedance and arithmetic operation capability).

In this paper, a new precision full-wave rectifier circuit using only two DDCCs is presented. Compared with previous rectifiers, the proposed structure is more suitable for integrated circuit fabrication. The circuit also offers a low output impedance terminal, which is suitable for low impedance load. Simulation results verifying the theoretical analysis are also included. To demonstrate the advantages of proposed configuration, pseudo RMS- 
to-DC conversion is also introduced.

\section{Circuit Realization}

The electrical symbol of DDCC is shown in Figure 1. The DDCC has three voltage input terminals: $Y_{1}, Y_{2}$ and $Y_{3}$, which have high input impedance. The terminal $X$ is a low impedance input terminal. There is a high impedance current output terminal $Z$. The CMOS realization for DDCC is shown in Figure 2 [11]. The input-output characteristics of the ideal DDCC is described as

$$
\left(\begin{array}{l}
V_{X} \\
I_{Y 1} \\
I_{Y 2} \\
I_{Y 3} \\
I_{Z}
\end{array}\right)=\left(\begin{array}{cccc}
1 & -1 & 1 & 0 \\
0 & 0 & 0 & 0 \\
0 & 0 & 0 & 0 \\
0 & 0 & 0 & 0 \\
0 & 0 & 0 & 1
\end{array}\right)\left(\begin{array}{c}
V_{Y 1} \\
V_{Y 2} \\
V_{Y 3} \\
I_{X}
\end{array}\right)
$$

The proposed full-wave rectifier circuit is shown in Figure 3. The circuit employs only two DDCCs. Compared to previous rectifiers, the proposed rectifier is higher suitable for IC implementation. The $\mathrm{DDCC}_{1}$ and $\mathrm{DDCC}_{2}$ are operated as non-inverting and inverting unity-gain voltage buffers. The input voltage $V_{\text {in }}$ is connected to $Y_{1}$ and $Y_{2}$ terminals of $\mathrm{DDCC}_{1}$ and $\mathrm{DDCC}_{2}$, respectively, while two outputs ( $X$ terminals) are connected. In this case, only positive peak will be appeared at the output voltage $V_{\text {out }}$.

The operation of the proposed full-wave rectifier is as follows: when $V_{\text {in }}>0$, the $\mathrm{DDCC}_{1}$ is on, the voltage $V_{\text {in }}$ is followed by the $\mathrm{DDCC}_{1}$ to the voltage $V_{\text {out }}$ at $X$ terminal. In addition, when $V_{\text {in }}<0$, the voltage $-V_{\text {in }}$ is followed by the $\mathrm{DDCC}_{2}$ to the voltage $V_{\text {out }}$ at $\mathrm{X}$ terminal $\left(-V_{Y 2}=V_{X}\right)$. From the operation of the proposed fullwave rectifier explained, the relations between the input voltage, $V_{\text {in }}$, and the output voltage, $V_{\text {out }}$, can be expressed as

$$
\left.\begin{array}{l}
V_{\text {in }}>0 ; V_{\text {out }}=V_{\text {in }}: \mathrm{DDCC}_{1}=\text { on } \\
V_{\text {in }}<0 ; V_{\text {out }}=-V_{\text {in }}: \mathrm{DDCC}_{2}=\text { on }
\end{array}\right\}
$$

The output voltage of the circuit in Figure 3 can be expressed as

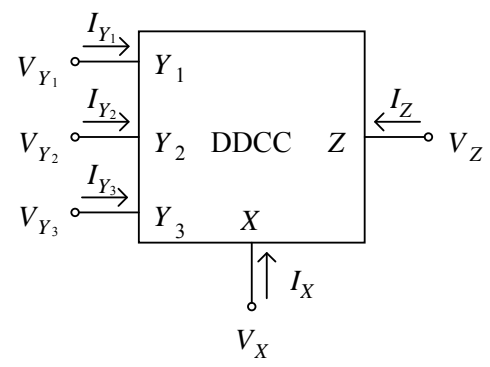

Figure 1. Electrical symbol for DDCC.

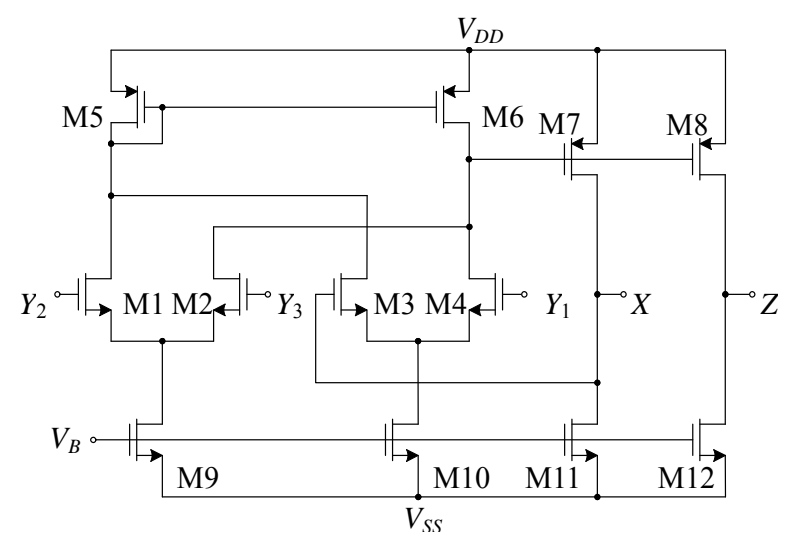

Figure 2. CMOS implementation for DDCC.

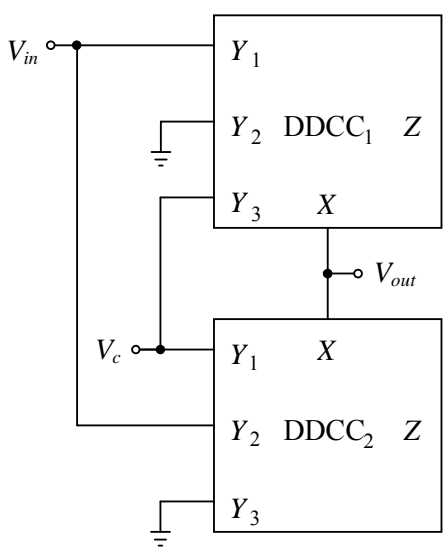

Figure 3. Proposed full-wave rectifier using DDCCs.

$$
V_{\text {out }}=\left|V_{\text {in }}\right|
$$

Therefore, the proposed circuit provides the full-wave rectification. It can be noted that the proposed circuit has high-input and low-output terminal, hence it is easy to drive loads without using a buffering device. The DC offset output voltage can be controlled by adjusting the voltage $V_{C}$.

\section{Simulation Results}

The proposed full-wave rectifier is simulated using PSPICE program to verify the given theoretical analysis. The DDCCs are simulated using CMOS structure of Figure 2 that can be implemented using CMOS structures DDCC given in [12]. The aspect ratios of the MOS transistors of the CMOS DDCC are given in Table 1.

The device model parameters used for the PSPICE simulation are taken from MIETEC $0.5 \mu \mathrm{m}$ CMOS process [12]. The supply voltages are selected as $V_{D D}=$ $-V_{S S}=2.5 \mathrm{~V}$ and the bias voltage is set as $V_{B}=-1.7 \mathrm{~V}$. The DC transfer characteristic of the proposed full-wave rectifier is shown in Figure 4, which shows the operating 
voltage ranging from $-1 \mathrm{~V}$ to $1 \mathrm{~V}$ of the input voltage. The magnified zero crossing of Figure 4 is shown in Figure 5. In this figure, the blunting region (b) is found as $-2.5 \mathrm{mV}<V_{\text {in }}<2.5 \mathrm{mV}$. Applying the $200 \mathrm{~m} V_{\text {peak }}$ sine wave at the input of the proposed rectifier, the input and output signals at frequencies of $500 \mathrm{kHz}$ and $1 \mathrm{MHz}$ are shown in Figures 6 and 7, respectively. This results is confirms the operation that the proposed rectifier can provide the full-wave rectification at the input signal amplitude lower than the threshold voltage of diode (< $0.3 \mathrm{~V}$ ). It is evident from Figures 6 and 7 that undistorted full-wave rectified signals are produced at all two frequencies. However, as the input frequency increases to $1 \mathrm{MHz}$ and beyond, the output signals have errors at the crossover region, called "corner distortion". This corner distortion results from the non-conduction/conction transition problem of DDCCs. At the frequency of $1 \mathrm{MHz}$, we simulate the temperature performance of the proposed full-wave rectifier as shown in Figure 3 by changing temperatures from $50^{\circ} \mathrm{C}$ to $100^{\circ} \mathrm{C}$. Figure 8 shows the output waveform of the proposed rectifier at temperatures of $50^{\circ} \mathrm{C}, 75^{\circ} \mathrm{C}$ and $100^{\circ} \mathrm{C}$. From simulation results in Figure 8, they show that the proposed circuit provides excellence temperature stability. This result is confirmed by Equation (3). The simulated peak outputs $V_{\text {out }}$ for the circuit were $199.46 \mathrm{mV}$ and $196.67 \mathrm{mV}$ at $50^{\circ} \mathrm{C}$ and $100^{\circ} \mathrm{C}$, respectively.

Table 1. Transistor aspect ratios of the used DDCC.

\begin{tabular}{ccc}
\hline Transistor & $W(\mu \mathrm{m})$ & $L(\mu \mathrm{m})$ \\
\hline M1-M4 & 1.6 & 1 \\
M5-M6 & 8 & 1 \\
M7-M9 & 20 & 1 \\
M10-M11 & 29 & 1 \\
M12-M14 & 90 & 1 \\
\hline
\end{tabular}

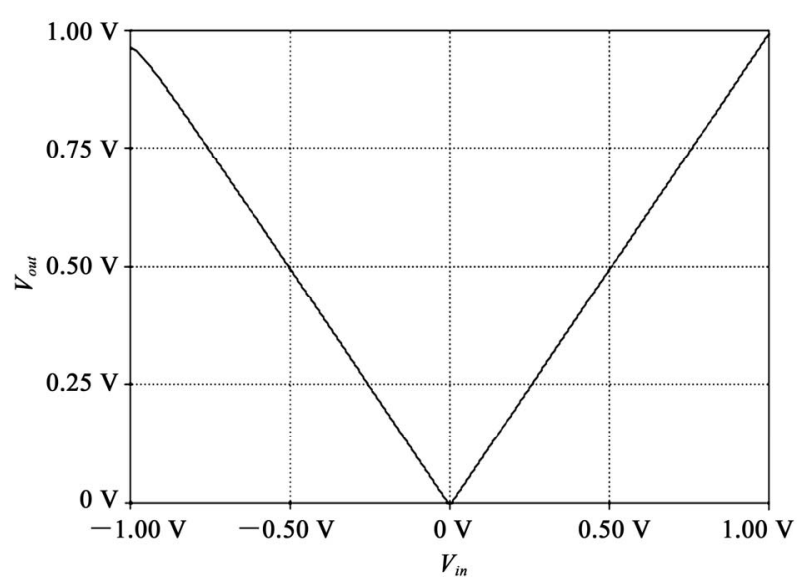

Figure 4. Simulated results for DC transfer characteristic.

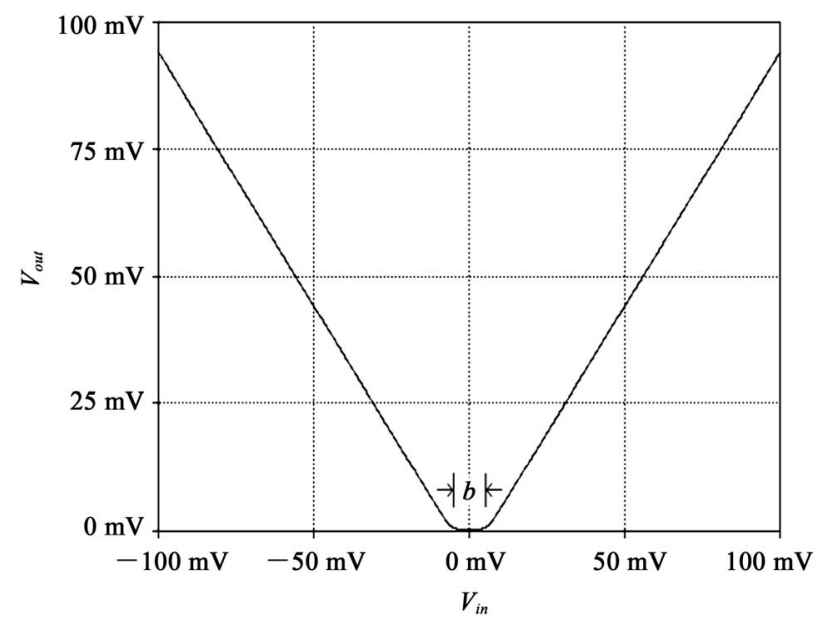

Figure 5. Simulated results for DC transfer characteristic at zero crossing regions.

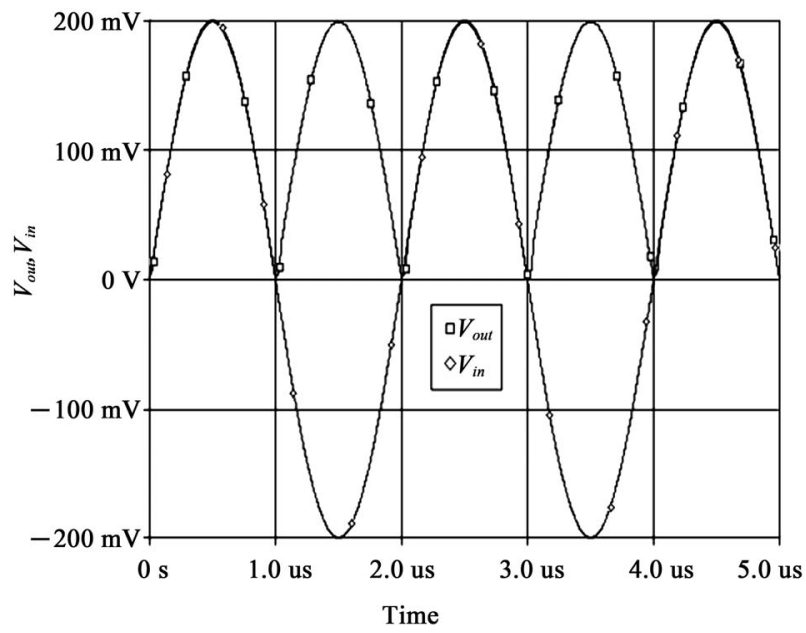

Figure 6. Operation of the proposed full-wave rectifier at $500 \mathrm{kHz}$ frequency for $V_{\text {in }}=200 \mathrm{mV}$ peak.

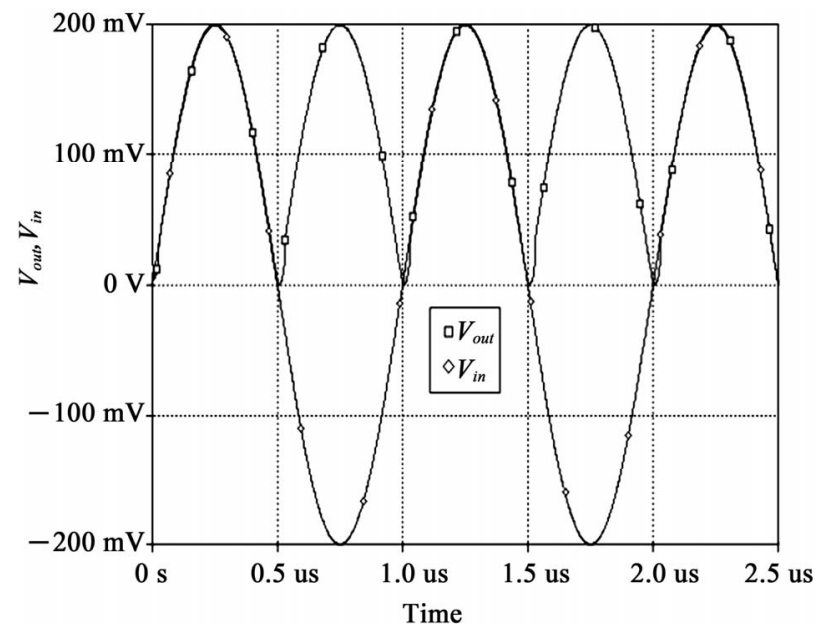

Figure 7. Operation of the proposed full-wave rectifier at $1 \mathrm{MHz}$ frequency for $V_{\text {in }}=200 \mathrm{mV}$ peak. 


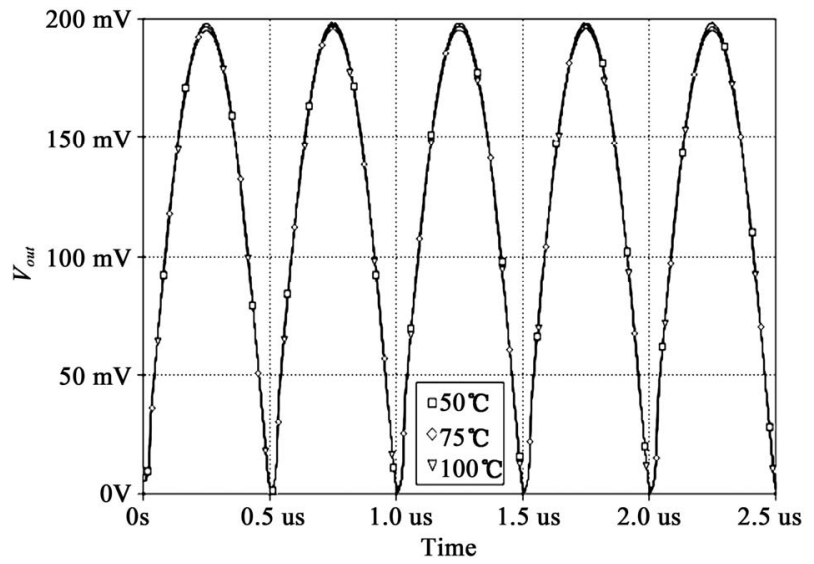

Figure 8. Operation of the proposed full-wave rectifier at different temperatures.

\section{Application Examples}

To show the advantage of proposed rectifier, author describes a pseudo root-mean-square (RMS)-to-DC conversion as an example. The well-known average value of a signal magnitude is defined by

$$
V_{\text {avg }}=\frac{1}{T} \int_{0}^{T}|v(t)| \mathrm{d} t
$$

where $v(t)$ is the AC signal, T is its period, and $V_{\text {avg }}$ is the average value of the rectified signal of $v(t)$. To perform this operation, the $\mathrm{AC}$ signal is first full-wave rectified and then low-pass filtered to extract the DC component. For the case of a sinusoidal signal we have $v(t)=V_{m}$ $\sin (2 \pi f t)$, where $V_{m}$ is the peak amplitude voltage and $f$ $=1 / T$ is the frequency. Substituting $v(t)$ into (4) and integrating yields

$$
V_{\text {avg }}=\frac{2}{\pi} V_{m}=0.637 V_{m}
$$

It is customary to calibrate the averaging circuits so that, with a sinusoidal input, the rms value is yield:

$$
V_{r m s}=\sqrt{\frac{1}{T} \int_{0}^{T} v^{2}(t) \mathrm{d} t}
$$

Substituting $v(t)=V_{m} \sin (2 \pi f t)$ and solving yields

$$
V_{r m s}=\frac{1}{\sqrt{2}} V_{m}=0.707 V_{m}
$$

Comparing (5) and (7) we have [13]

$$
\frac{V_{\text {rms }}}{V_{\text {avg }}}=\frac{\pi}{2 \sqrt{2}} \approx 1.111
$$

which is the amount of amplification required to obtain $V_{r m s}$ from $V_{\text {avg }}$. By using proposed full-wave rectifier, both average value and RMS value of sinusoidal waveform can be readily implemented as shown in Figure 9. It should be noted that only grounded components are required. For more suitable IC implementation, all grounded resistors can be replaced by MOS resistor as shown in Figure 10. This resistor was presented in 1990 by Wang [14]. Assume $\mathrm{MR}_{1}$ and $\mathrm{MR}_{2}$ have the same characteristics and remaining in the saturation region. The resistance value of MOS resistor can be expressed as:

$$
R_{e q}=\frac{1}{2 K\left(V_{D D}-V_{T H}\right)}
$$

where $K=\mu_{\mathrm{o}} \mathrm{C}_{\mathrm{ox}}(W / L), \mathrm{V}_{\mathrm{TH}}$ is the threshold voltage and $V_{D D}=\left|V_{S S}\right|$ are the supply voltages. It can see that the value of resistor can vary by setting the appropriate aspect ratio.

A first-order low-pass filter is achieved with a properly specified $R_{L}$ and $C$. The voltage $V_{\text {out }}$ is converted to the input current of the filter in which the AC components are filtered through the capacitor $C$. The voltage value, $V_{\text {avg }}$, can be obtained by choosing $R_{i n}$ and $R_{L 1}$ identical. According to (8), $V_{r m s}$ can be obtained by setting $R_{L 2} / R_{\text {in }}=1.111$. For a symmetrical square input, the ratio of (8) becomes unity thus $V_{\text {avg }}$ of Figure 9 gives the exact RMS value because $R_{i n}$ and $R_{L 1}$ are identical. If $R_{L 1} / R_{\text {in }}=1.155$, the circuit may indicate the RMS value for either sinusoidal or triangular current signals. The criterion for specifying $C_{L}$ (i.e. $C_{L}=C_{L 1}=C_{L 2}$ ) is that it must be large enough to keep the residual output ripple within specified limits, that is [13]

$$
C_{L} \geq \frac{1}{4 \pi f_{\min }}
$$

where $f_{\min }$ is the low end of the frequency range of interest. $C_{L}$ should usually exceed the right-hand term by the inverse of the fractional ripple error that can be tolerated at the output [14].

The RMS-to-DC converter in Figure 9 is simulated using PSPICE program to verify the given theoretical analysis. For this simulation, three resistors $\left(R_{i n}, R_{L 1}\right.$ and

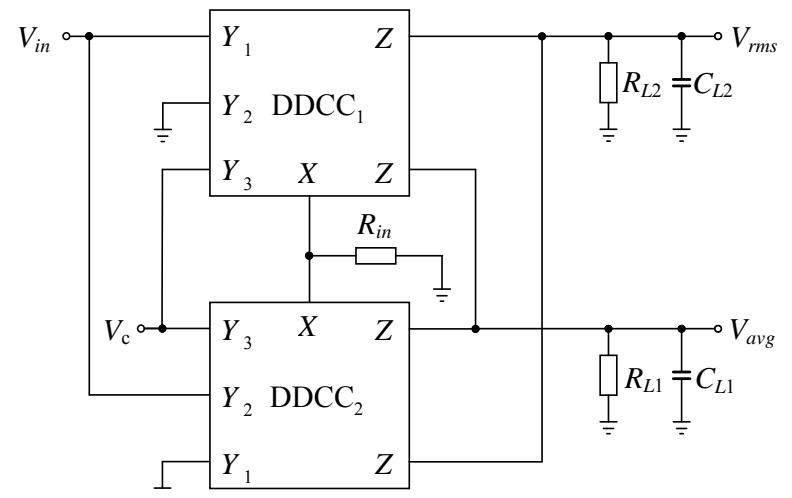

Figure 9. The full-wave rectifier-based RMS-to-DC converter for sinusoidal waveform. 
$R_{L 2}$ ) are replaced by MOS resistor as shown in Figure 10 According to (8), when $R_{i n}$ and $R_{L 1}$ are identical to obtain $V_{a v}$, then $V_{r m s}$ can be obtained by setting $R_{L 2} / R_{i n}=1.111$. In this case, the aspect ratios (W/L) of MOS transistors, $\mathrm{MR}_{1}$ and $\mathrm{MR}_{2}$, for $R_{i n}, R_{L 1}$ and $R_{L 2}$ are $2 \mu \mathrm{m} / 2 \mu \mathrm{m}, 2 \mu \mathrm{m} /$ $2 \mu \mathrm{m}$ and $1.8 \mu \mathrm{m} / 2 \mu \mathrm{m}$, respectively $\left(R_{i n}=R_{L 1}=3.457 \mathrm{k} \Omega\right.$ and $R_{L 2}=3.844 \mathrm{k} \Omega$ ). A sinusoidal input with $V_{i n}=200 \mathrm{mV}$ and $f=1 \mathrm{MHz}$ was applied to the converter and $C=1 \mathrm{nF}$. The supply voltages for DDCC are selected as $V_{D D}=$ $-V_{S S}=2.5 \mathrm{~V}$ and the bias voltage is set as $V_{B}=-1.7 \mathrm{~V}$. The multiple-output DDCC can be obtained by adding additional current mirrors. Figure 11 shows the simulation results for $V_{a v g}=126.63 \mathrm{mV}$ and $V_{r m s}=140.45 \mathrm{mV}$, with ripple less than $2 \%$. In this case, the RMS voltage converter for both sinusoidal (output $V_{r m s}$ ) and symmetrical square (output $V_{\text {avg }}$ ) waveform can be obtained. To achieve this case, the buffer circuits should be used.

\section{Conclusions}

In this paper, a new DDCC-based full-wave rectifier has been presented. The proposed circuit comprises only two DDCC which is suitable for IC implementation. Theproposed circuit has high-input and low-output imped-

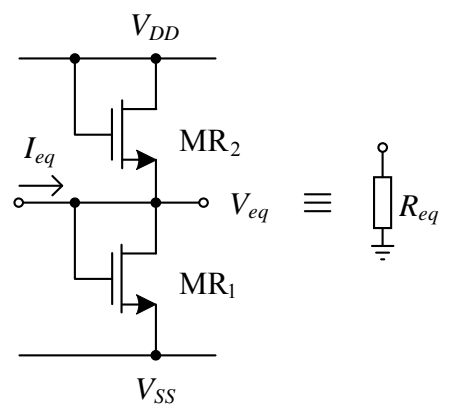

Figure 10. MOS resistor.

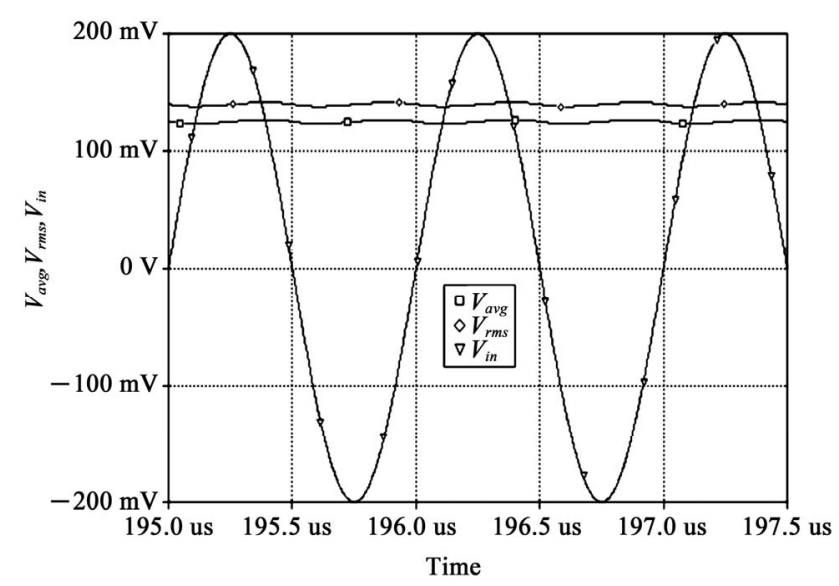

Figure 11. Simulated $V_{a v g}$ and $V_{r m s}$ for sinusoidal input with amplitude of $200 V_{\text {Peak }}$ and frequency of $1 \mathrm{MHz}$. ance terminals, hence it easy to drive loads without using a buffering device. It can be applied to various nonlinear analog signal processing circuits. The performance of the proposed circuit is confirmed from PSPICE simulation results. Simulation results show that the proposed rectifier have excellent temperature stability. The application of proposed rectifier to pseudo RMS-to-DC conversion is also included.

\section{References}

[1] J. Peyton and V. Walsh, "Analog Electronics with Op Amps: A Source Book of Practical Circuits," Cambridge University Press, New York, 1993.

[2] R. G. Irvine, "Operational Amplifier Characteristics and Applications," Prentice Hall International, Upper Saddle River, 1994.

[3] Z. Wang, "Full-Wave Precision Rectification That is Performed in Current Domain and Very Suitable for CMOS Implementation," IEEE Transactions on Circuits and Systems-I, Vol. 39, No. 6, 1992, pp. 456-462. doi:10.1109/81.153637

[4] S. J. G. Gift, "A High-Performance Full-Wave Rectifier Circuit," International Journal of Electronics, Vol. 89, No. 8, 2000, pp. 467-476.

[5] C. Toumazou, F. J. Lidgey and S. Chattong, "High Frequency Current Conveyor Precision Full-Wave Rectifier," Electronics Letters, Vol. 30, No. 10, 1994, pp. 745746. doi:10.1049/el:19940539

[6] A. Khan, M. A. El-Ela and M. A. Al-Turaigi, "CurrentMode Precision Rectification," International Journal of Electronics, Vol. 79, No. 6, 1995, pp. 853-859. doi:10.1080/00207219508926319

[7] K. Hayatleh, S. Porta and F. J. Lidgey, "Temperature Independent Current Conveyor Precision Rectifier," Electronics Letters, Vol. 30, No. 25, 1995, pp. 2091-2093. doi:10.1049/el:19941454

[8] A. Monpapassorn, K. Dejhan and F. Cheevasuvit, "A FullWave Rectifier Using a Current Conveyor and Current Mirrors," International Journal of Electronics, Vol. 88, No. 7, 2001, pp. 751-758. doi:10.1080/00207210110052892

[9] W. Surakumpontorn, K. Anuntahirunrat and V. Riewruja, "Sinusoidal Frequency Doubler and Full-Wave Rectifier Using Translinear Current Conveyor," Electronics Letters, Vol. 34, No. 22, 1998, pp. 2077-2079. doi:10.1049/el:19981456

[10] E. Yuce, S. Minaei and O. Cicekoglu, "Full-Wave Rectifier Realization Using Only Two CCII+S and NMOS Transistors," International Journal of Electronics, Vol. 93, No. 8, 2006, pp. 533-541. doi:10.1080/00207210600711606

[11] W. Chiu, S.-I. Liu, H.-W. Tsao and J.-J. Chen, "CMOS Differential Difference Current Conveyors and Their Applications," IEE Proceeding of Circuits, Devices, System, Vol. 143, No. 2, 1996, pp. 91-96. doi:10.1049/ip-cds: 19960223 
[12] S. Minaei and M. A. Ibrahim, "General Configuration for Realizing Current-Mode First-Order All-Pass Filter Using DDCC," International Journal of Electronics, Vol. 92, No. 6, 2005, pp. 347-356.

doi:10.1080/00207210412331334798

[13] Z. Wang, "Novel Pseudo RMS Current Converter for Sinusoidal Signals Using a CMOS Precision Current
Rectifier," IEEE Transactions on Instrumentation and Measurement, Vol. 39, No. 4, 1990, pp. 670-671. doi:10.1109/19.57256

[14] Z. Wang, "2-MOSFET Transistor with Extremely Low Distortion for Output Reaching Supply Voltage," Electronics Letters, Vol. 26, No. 13, 1990, pp. 951-952. doi:10.1049/el:19900620 